\title{
Alterações fisiológicas e metabólicas em indivíduo com distrofia muscular de Duchenne durante tratamento fisioterapêutico: um estudo de caso
}

\author{
Physiological and metabolic alterations in Duchenne's muscular dystrophy \\ patient during a physiotherapeutic treatment: a case study
}

\author{
Monique da Silva Gevaerd ${ }^{[a]}$, Susana Cristina Domenech ${ }^{[b]}$, Noé Gomes Borges Júnior ${ }^{[c]}$, \\ Daniela Fernanda Higa ${ }^{[\mathrm{d}]}$, Adriano Eduardo Lima-Silva ${ }^{[\mathrm{e}]}$
}

[a] Doutora em Farmacologia pela Universidade do Estado de Santa Catarinam (UDESC), Centro de Ciências da Saúde e do Esporte (CEFID), Laboratório de Análises Multisetorial (MULTILAB), Florianópolis, SC - Brasil, e-mail: moniquegevaerd@yahoo.com.br

[b] Doutora em Ciências e Engenharia de Materiais pela Universidade do Estado de Santa Catarina (UDESC), Centro de Ciências da Saúde e do Esporte (CEFID), Laboratório de Instrumentação (LABIN), Florianópolis, SC - Brasil, e-mail: scdomenech@gmail.com

[c] Doutor em Biofísica pela Universidade do Estado de Santa Catarina (UDESC), Centro de Ciências da Saúde e do Esporte (CEFID), Laboratório de Instrumentação (LABIN), Florianópolis, SC - Brasil, e-mail: d2ngbj@udesc.br

[d] Especialista intensiva em Fisioterapia pela Universidade do Estado de Santa Catarina (UDESC), Centro de Ciências da Saúde e do Esporte (CEFID), Curso de Fisioterapia, Florianópolis, SC - Brasil, e-mail: danifehiga@yahoo.com.br

[e] Doutor em Educação Física pela Universidade Federal de Alagoas (UFAL), Laboratório de Aptidão Física, Desempenho e Saúde, Grupo de Pesquisa em Ciências do Esporte (GPCE), Maceió, AL - Brasil, e-mail: limasilvaae@hotmail.com

\begin{abstract}
Resumo
Introdução: A Distrofia Muscular de Duchenne (DMD) caracteriza-se por uma perda progressiva da força muscular. O tratamento fisioterapêutico tem fundamental importância na manutenção da qualidade de vida desses pacientes. Contudo, existe grande dificuldade em graduar o grau de cansaço e o limite de exigência da musculatura durante o tratamento. Adicionalmente, não existe na literatura descrição sobre alterações fisiológicas e metabólicas durante uma atividade de esforço nessa população específica. Objetivos: O presente trabalho teve como objetivos avaliar as alterações fisiológicas e metabólicas em indivíduo com DMD durante um programa de fisioterapia, relacionando-as com a percepção subjetiva de esforço (PSE). Metodologia: Este estudo foi realizado na Clínica de Prevenção e Reabilitação Física da Universidade do Estado de Santa Catarina (UDESC). O sujeito do estudo foi um paciente com DMD (sexo masculino, 17 anos). Foram coletados dados de concentrações plasmáticas de lactato e glicose, frequência cardíaca e frequência respiratória, pressão arterial e percepção de esforço segundo a escala de PSE CR10 de Borg. Os dados foram coletados aos $0,15,30$ e 45 minutos de atendimento fisioterapêutico. A sessão de fisioterapia foi dividida em 3 etapas com atividade de intensidade e exigência muscular crescentes. Foram realizados 6 atendimentos. Resultados: Os resultados demonstraram PSE crescente durante a sessão de fisioterapia. Foi verificada uma relação linear entre as variáveis metabólicas e a PSE. Já
\end{abstract}


as variáveis fisiológicas não demonstraram relação proporcional com a PSE. Conclusão: Sugerese que a PSE é uma forma simples de monitoramento do cansaço de pacientes com DMD, durante o tratamento fisioterapêutico.

Palavras-chave: Distrofia muscular de Duchenne. Alterações metabólicas e fisiológicas. Percepção subjetiva de esforço.

\begin{abstract}
Introduction: Duchenne muscular dystrophy (DMD) is the most common and severe childhood muscular dystrophy, resulting in progressive muscle weakness. Physiotherapeutic treatment is essential to enhance the patient's quality of life. However, it is difficult to graduate the fatigue of the patient during treatment. Also, there is no description about physiological and metabolic changes during an activity of effort in DMD patients. Objectives: The purpose of the present study was to evaluate the physiological and metabolic changes in DMD patient during a physiotherapeutic treatment, relating them to the ratings of perceived exertion (PSE). Methods: The study subject was a patient with DMD (male, 17 years). Were collected data on plasma concentrations of glucose and lactate, heart rate, respiratory rate, blood pressure and ratings of perceived exertion using Borg's CR10 scale. Data were collected to: 0, 15, 30 and 45 minutes of physiotherapeutic treatment. The session of physiotherapy was divided into 3 steps with increasing intensity of activity and muscular demand. Results: The results showed a growing PSE during the physiotherapeutic treatment. It was found a proportional relationship between the PSE and metabolic variables. The physiological variables showed no proportional relationship with the PSE. Conclusion: Finally, it is suggested that the PSE is a simple way of monitoring the patient's fatigue with $D M D$, during physiotherapeutic treatment.
\end{abstract}

Keywords: Muscular dystrophy of Duchenne. Physiological and metabolic changes. Ratings of perceived exertion.

\title{
Introdução
}

As distrofias compreendem um grupo de miopatias caracterizadas pela degeneração progressiva da musculatura estriada. Dentre elas, a mais frequente é a Distrofia Muscular de Duchenne (DMD), com incidência de 1 caso entre 3.500 nascidos vivos do sexo masculino (1-5).

A DMD é um distúrbio recessivo ligado ao cromossomo $\mathrm{X}$, causada por mutação no gene que codifica a distrofina, uma proteína localizada no sarcolema das fibras musculares. Os pacientes com DMD têm importante deficiência dessa proteína, apresentando menos que 3\% do total que apresenta um indivíduo normal. A deficiência da distrofina é um dos fatores iniciais nas lesões das membranas das fibras musculares, permitindo maior ingresso de cálcio intracelular e ativação de proteases, determinando a necrose das fibras, fagocitose, reações inflamatórias e posterior proliferação de tecido conjuntivo e infiltração adiposa (6-10).

As manifestações clínicas da DMD estão presentes desde o nascimento, mas tornam-se evidentes entre os 3 e 5 anos de idade. A principal característica clínica é a perda progressiva da força muscular, com predileção pelos músculos proximais dos membros e pelos músculos flexores do pescoço. Com o decorrer do tempo, os pacientes perdem a capacidade de deambulação, apresentam sinais de fraqueza muscular e sensação de cansaço ao realizar uma atividade de esforço. Aos 12 anos de idade, a maioria dos pacientes está confinada à cadeira de rodas $(1-5,8)$. 
Apesar de ser uma doença incurável, é necessário que o tratamento desses pacientes seja multidisciplinar visando sempre o seu bem-estar. Dentre os tratamentos utilizados, a fisioterapia apresenta importância fundamental para a prevenção de complicações respiratórias e de contraturas, para a manutenção da força muscular e da mobilidade, visando a promoção de independência para as atividades de vida diárias e, consequentemente, para a melhoria da qualidade de vida desses pacientes. No entanto, é importante que o tratamento fisioterapêutico tenha a preocupação de não fadigar o paciente, visto que o excesso de exigência muscular leva a uma aceleração no processo degenerativo (11-16).

Contudo, existem dificuldades para alcançar esses objetivos, pelas limitações do paciente com DMD, principalmente em função da fraqueza muscular. Além disso, os métodos empregados na reabilitação são bastante restritos, por causa do cuidado para não fadigar o paciente. Há, ainda, uma grande dificuldade, principalmente em pacientes com a doença mais evoluída, em graduar o grau de cansaço, bem como o limite de exigência da musculatura durante o tratamento (11-16).

Por outro lado, sabe-se que durante uma atividade de esforço, o organismo reage modificando algumas variáveis fisiológicas e metabólicas para permitir a liberação de energia necessária para a manutenção da atividade e da força. Variáveis metabólicas como concentração sanguínea de lactato [LA] e glicose [GLI] são medidas importantes para a determinação do grau de intensidade da atividade e da fadiga. Além disso, alterações fisiológicas como pressão arterial (PA), frequência cardíaca (FC) e frequência respiratória (FR) também são parâmetros que revelam a tentativa do organismo em suprir as necessidades de oxigênio e de nutrientes, que aumentam durante o exercício (17-23).

Essas alterações metabólicas e fisiológicas que ocorrem durante uma atividade de esforço refletem no sujeito em atividade sensação de cansaço e fadiga. Essa percepção subjetiva de esforço (PSE) revela a sensação do sujeito em relação ao exercício realizado. A PSE também é uma medida muito utilizada em treinamentos de atletas verificando a intensidade dos exercícios $(24,25)$.

Constata-se, entretanto, que apesar do grande número de pesquisas relacionadas à fisiologia do exercício, poucos estudos têm avaliado as alterações do organismo durante atividade de esforço em grupos de pacientes com doenças associadas. Da mesma forma, as pesquisas com pacientes com distrofias visam, em sua maioria, a procedimentos terapêuticos e diagnósticos. Assim, existem poucos estudos relacionando essas duas áreas. Diante do exposto, o presente estudo teve como objetivo realizar um estudo de caso para avaliar as alterações fisiológicas (PA, FC e FR) e metabólicas [LA] e [GLI] em um indivíduo com DMD, durante um programa de fisioterapia, relacionando-as com a PSE.

\section{Materiais e métodos}

\section{Relato de caso}

O estudo foi desenvolvido na Clínica de Prevenção e Reabilitação Física da Universidade do Estado de Santa Catarina (UDESC), com um indivíduo de 17 anos, do sexo masculino, com diagnóstico de DMD. O voluntário apresentou os primeiros sintomas, como quedas constantes, sensação de fraqueza e dificuldades para deambular, aos 4 anos de idade. Aos 5 anos foi diagnosticada a DMD. Aos 10 anos a criança não conseguia mais deambular sem auxílio e começou a utilizar a cadeira de rodas. Com essa idade, iniciou o tratamento fisioterapêutico.

Antes de iniciar a pesquisa, o voluntário e seu responsável foram devidamente informados sobre todos os procedimentos a serem realizados. Após concordarem em participar do estudo, o responsável assinou um termo de consentimento aprovado pelo Comitê de Ética em Pesquisa em Seres Humanos da UDESC (protocolo n. 020/2005). 


\section{Procedimentos para coleta de dados}

Foram avaliadas as seguintes variáveis no presente estudo:

- Frequência respiratória (FR): obtida por meio da mensuração manual com auxílio de estetoscópio.

- Frequência cardíaca (FC): obtida por meio do Monitor de Batimentos Cardíacos - Polar 610 i.

- Pressão Arterial (PA): obtida por meio do monitor automático de pressão arterial CITIZEN CH 656 C.

- Concentrações sanguíneas de Lactato [LA]: mensuradas por meio de microamostras de sangue retirado da extremidade distal do lóbulo da orelha externa, sendo a análise feita em lactímetro Yelow Spring® (modelo 1500).

- Concentrações sanguíneas de Glicose [GLI]: mensuradas com auxílio do glicosímetro portátil Accu-Chek, utilizando microamostras $(25 \mathrm{ml})$ de sangue retirado da extremidade das falanges distais da mão.

- Percepção Subjetiva de Esforço (PSE): obtida do indivíduo com a visualização da escala CR10 de Borg.

Ao chegar à clínica, o voluntário recebeu explicações sobre a graduação da escala de PSE e demais instrumentos a serem utilizados. Em seguida, foram mensurados os dados de PSE, FC, FR, PA, [LA] e [GLI] de repouso. Neste momento, o indivíduo encontrava-se em decúbito dorsal. Logo após, foi iniciada a sessão de fisioterapia, sendo esta dividida em três partes: nos intervalos de 0 a 15 minutos; 15 a 30 minutos e 30 a 45 minutos. Cada fase teve procedimentos e objetivos distintos, visando às diferentes debilidades apresentadas pelo voluntário.

$\mathrm{Na}$ primeira parte da sessão, 15 minutos iniciais, foram realizadas mobilizações passivas das articulações e alongamento da musculatura dos membros superiores e inferiores; alongamento da musculatura acessória da respiração; massoterapia e deslizamentos de fáscies na região plantar de ambos os tarsos e na musculatura posterior da perna. Essa primeira fase foi constituída de atividades passivas, ou seja, sem a necessidade de controle dos movimentos ou geração de força pelo indivíduo. Após os primeiros 15 minutos do atendimento fisioterapêutico, foi realizada a verificação das mesmas variáveis metabólicas e fisiológicas anteriormente citadas.

A segunda fase do tratamento fisioterapêutico visou à manutenção do condicionamento respiratório. O tratamento iniciou com controle da respiração com estimulação diafragmática proprioceptiva. Logo após, foram realizados exercícios de expansão torácica, com inspirações ativas, sustentação da inspiração e inspirações fracionadas. Para a realização dessa segunda etapa do tratamento fisioterapêutico, foi necessário o controle voluntário da respiração. Ao final dos 30' foram novamente realizadas as coletas dos dados.

$\mathrm{Na}$ terceira etapa, nos 15' finais da sessão fisioterapêutica, o indivíduo realizou movimentos ativos e ativos assistidos, com os membros superiores e inferiores. Foram utilizados bastões e bolas como objetos de auxílio e de incentivo. Nessa etapa, o indivíduo foi posicionado sentado com apoio dorsal e realizava, nessa postura, exercícios de flexo-extensão de cotovelos, ombros e joelhos. Foram realizadas aproximadamente 3 séries de 8 repetições, variando de acordo com o relato de cansaço do voluntário. Nessa fase final do atendimento, o controle do movimento e de força durante as atividades realizadas foi estabelecido pelo indivíduo. Ao término da sessão, o procedimento de coleta de dados foi novamente repetido.

Ao final do estudo, foram realizadas 6 sessões de fisioterapia, com intervalos de 1 ou 2 dias entre cada atendimento. Em cada sessão foram realizadas 4 coletas dos dados de FC, FR, PA, PSE, [LA] e [GLI], sendo uma em repouso e as demais ao final de cada fase da sessão fisioterapêutica. 


\section{Tratamento estatístico}

As comparações estatísticas dos resultados obtidos foram realizadas utilizando o software Statistica 6.0. Inicialmente foram feitas as médias dos tempos 0, 15, 30 e 45 minutos dos 6 dias de atendimento. Posteriormente, foi feito o Teste t de Student pareado entre as médias de todos os tempos $(0,15,30$ e 45 minutos). Para avaliar a correlação entre as variáveis, empregou-se o Teste de correlação de Spearman. Os dados foram expressos como a média \pm erro padrão da média (e.p.m.) e a probabilidade aceita como indicativa da existência de diferenças estatisticamente significativas foi $\mathrm{p} \leq 0.05$.

\section{Resultados}

\section{Percepção subjetiva de esforço (PSE)}

A PSE aumentou linearmente no decorrer da sessão de fisioterapia entre os intervalos 0’ e 45', sendo observada diferença significativa entre as médias de PSE em todos os intervalos avaliados (Figura 1). Por outro lado, a análise das médias da PSE de cada sessão não alterou significativamente em relação aos 6 dias de atendimento.

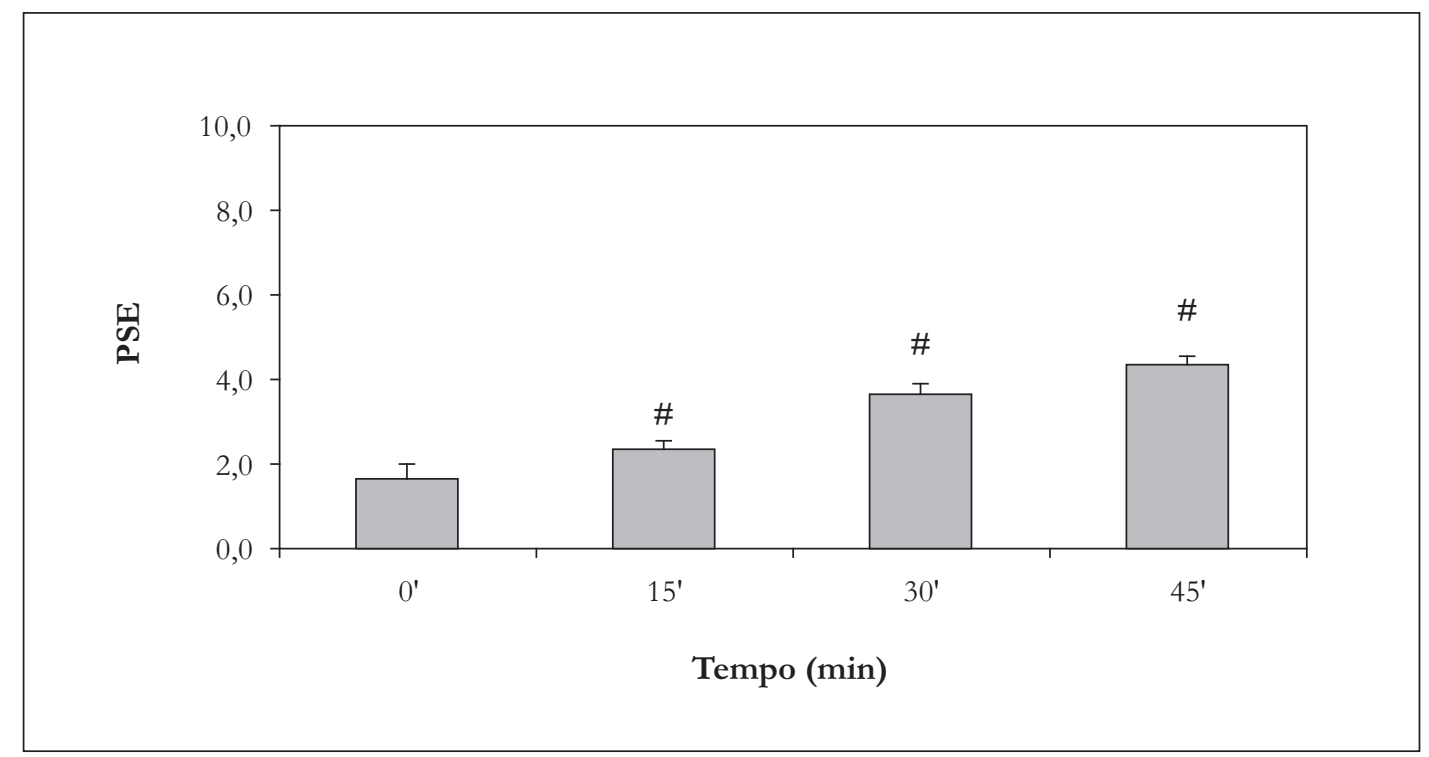

Figura 1 - Percepção subjetiva de esforço nos intervalos de 0 a 45 min. Os valores representam a média \pm e.p.m. das 6 sessões de fisioterapia. \# p $\leq 0,05$ em relação a todos os tempos analisados (Teste t de Student pareado)

\section{Concentrações plasmática de lactato ([LA])}

No presente estudo, apesar da [LA] não ter ultrapassado o valor de $1 \mathrm{mmol} / \mathrm{l}$, foi observado aumento linear das [LA] ao longo do desenvolvimento de cada sessão de fisioterapia, com diferença significativa entre os tempos 0 e 45 minutos (Figura 2). Não foi observada variação das [LA] entres os 6 dias de atendimento fisioterapêutico. 


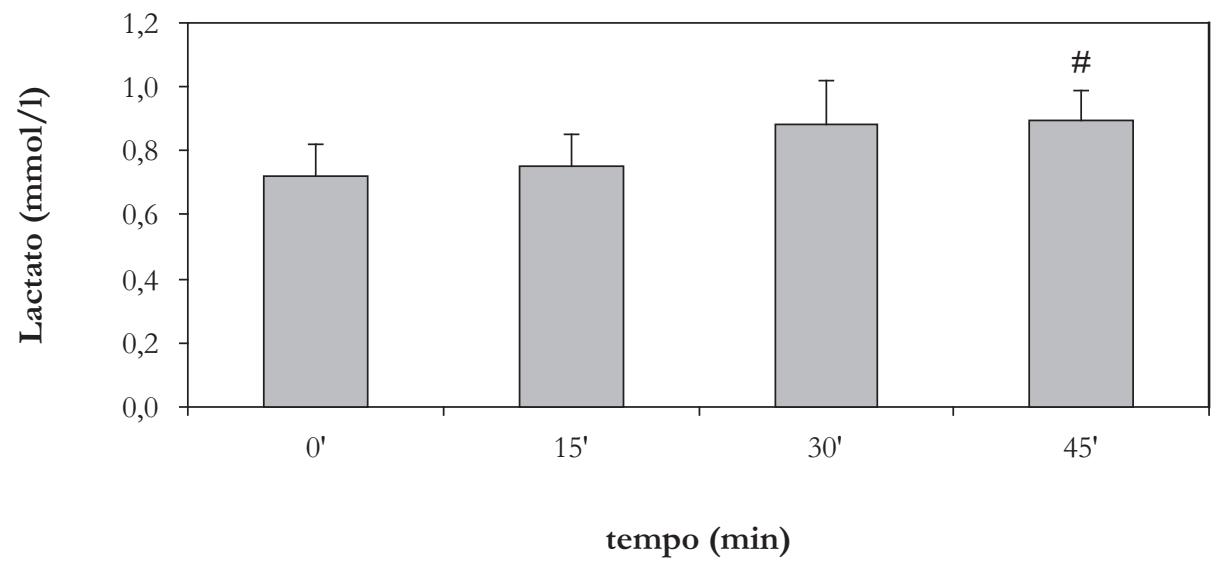

Figura 2 - Concentrações plasmáticas de lactato nos intervalos de 0 a 45 min. Os valores representam a média \pm e.p.m. das 6 sessões de fisioterapia. \# p $\leq 0,05$ em relação ao tempo 0' ('Teste t de Student pareado)

Além dos dados já relatos, foi verificado por meio do Teste de correlação de Spearman uma relação linear entre as médias da [LA] e da PSE no decorrer de cada sessão de fisioterapia (Spearman $\mathrm{r}=0,949 ; \mathrm{p}=0,026)$. Ou seja, observou-se que o aumento das [LA] foi diretamente proporcional à intensidade dos exercícios aplicados durante a sessão de fisioterapia (Figura 3).

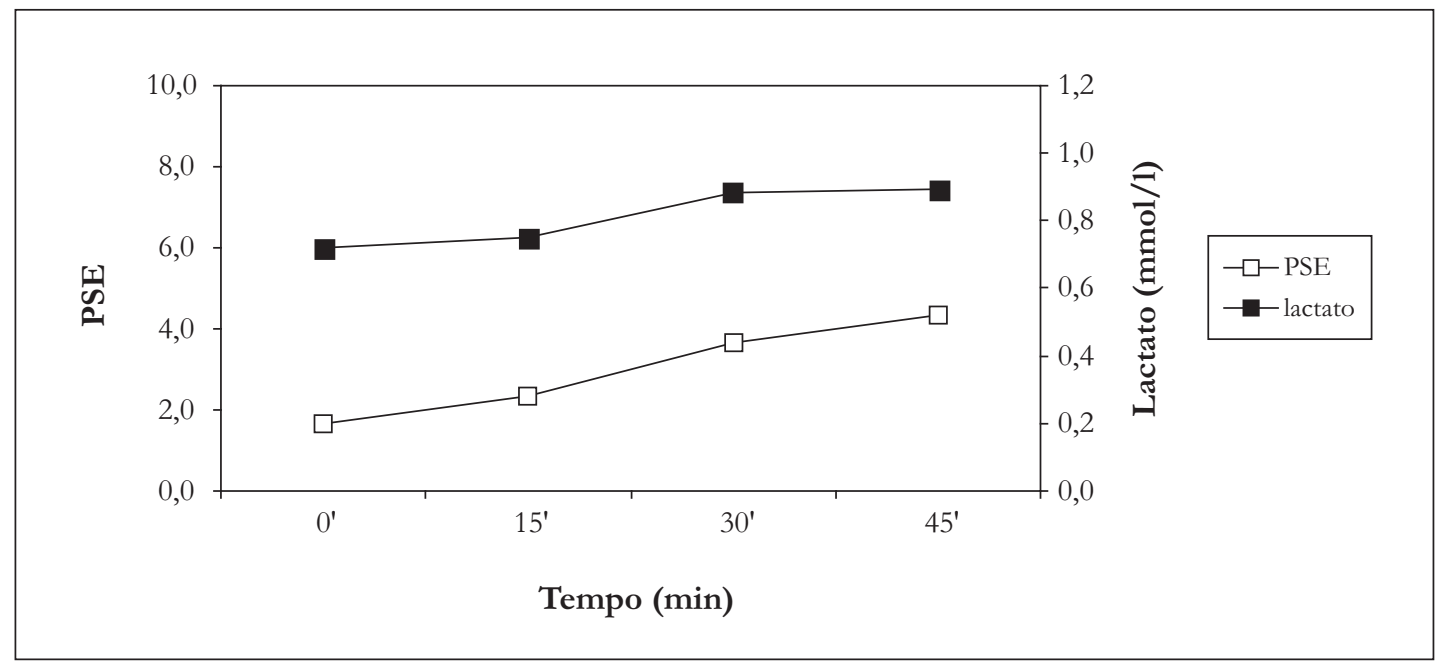

Figura 3 - Relação das médias de concentrações plasmáticas de lactato e PSE nos intervalos de 0 a 45 min. Os valores representam a média \pm e.p.m. das 6 sessões de fisioterapia

\section{Concentrações plasmática de glicose ([GLI])}

De acordo com os resultados obtidos, as [GLI] não variaram significativamente ao longo de cada sessão de fisioterapia (Figura 4). Dessa forma, não foi verificada relação entre as curvas de concentração plasmática de glicose e PSE. Já na comparação entre as 6 sessões de fisioterapia realizadas, foi verificada uma pequena variação das médias de [GLI]. 


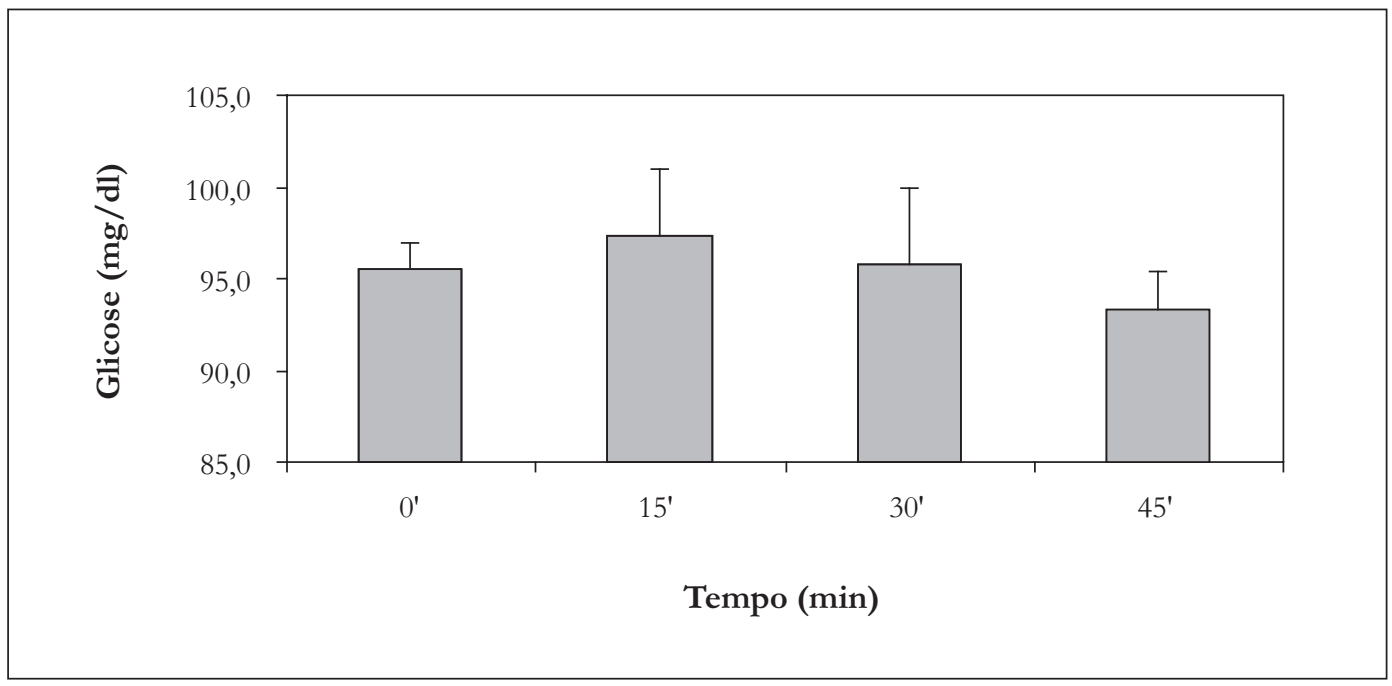

Figura 4 - Concentrações plasmáticas de glicose nos intervalos de 0 a 45 min. Os valores representam a média \pm e.p.m. das 6 sessões de fisioterapia. Não houve diferença estatística significativa entre os intervalos de tempo avaliados

\section{Frequência cardíaca (FC)}

No presente estudo não foi verificada variação significativa entre as médias de FC nos diferentes intervalos de tempo avaliados (Tabela 1). Consequentemente, não foi observada relação diretamente proporcional entre as FC apresentadas e a PSE relatada pelo indivíduo. Adicionalmente, no decorrer dos 6 dias de atendimento fisioterapêutico, foi verificada variação das médias de FC, sendo que esta ocorreu de forma aleatória.

Tabela 1 - Médias das variáveis fisiológicas nos intervalos de 0 a 45 min. das sessões de fisioterapia

\begin{tabular}{lllll}
\hline Variáveis Fisiológicas & Tempo 0, & Tempo 15, & Tempo 30' & Tempo 45' \\
\hline FC (bpm) & $106.3 \pm 5.18$ & $108.0 \pm 3.2$ & $105.8 \pm 3.4$ & $100.1 \pm 6.5$ \\
PAS (mmHg) & $87.0 \pm 2.5$ & $85.8 \pm 1.1$ & $82.8 \pm 1.4$ & $89.1 \pm 1.2 *$ \\
PAD (mmHg) & $58.5 \pm 4.1$ & $56.6 \pm 2.5$ & $57.6 \pm 1.6$ & $62.3 \pm 1.1 \#$ \\
FR (rpm) & $27.6 \pm 1.2$ & $30.6 \pm 1.2 *$ & $26.6 \pm 0.9$ & $31.0 \pm 0.85 *$ \\
\hline
\end{tabular}

Nota: Os valores representam a média \pm e.p.m. das 6 sessões de fisioterapia. ${ }^{*} \mathrm{p} \leq 0,05$ em relação ao tempo 30 ; \# $\mathrm{p} \leq 0,05$ em relação aos tempos 15' e 30'; * p $\leq 0,05$ em relação ao tempo 0’; $\mathrm{p} \leq 0,05$ em relação ao tempo 15' e 45' (Teste t de Student pareado)

\section{Pressão arterial sistólica (PAS) e pressão arterial diastólica (PAD)}

Os resultados demonstraram que o indivíduo apresentou decréscimo nas médias das PAS, seguido por aumento nos últimos 15 minutos de atendimento, sendo observada diferença significativa entre os tempos 30' e 45' (Tabela 1).

Já as médias de PAD não variaram de forma contínua entres os intervalos de tempo avaliados. Mas foi observada uma diferença significativa entre a média da PAD aos 45' em relação aos tempos 15' e 30' (Tabela 1).

Apesar dos valores finais de PAS e PAD apresentarem diferença significativa, as variações observadas não foram diretamente proporcionais às alterações da PSE. 


\section{Frequência Respiratória (FR)}

Os dados coletados referentes à FR, evidenciaram que esta variável não apresentou variação gradual com o decorrer da sessão de fisioterapia. Mas foi observada diferença significativa entre as médias dos tempos 15' e 45' em relação ao tempo 0; bem como entre a média da FR do tempo 30' em relação aos tempos 15' e 45' (Tabela 1). Adicionalmente, os resultados obtidos não evidenciaram uma relação diretamente proporcional entre as médias de FR e a PSE.

\section{Discussão}

\section{Percepção subjetiva de esforço (PSE)}

Segundo Borg $(24,25)$, o aumento progressivo e gradual da PSE, é geralmente observado em exercícios com intensidades continuamente aumentadas. Portanto, observamos que o tratamento fisioterapêutico proposto no presente estudo aumentou progressivamente a intensidade dos exercícios, apresentando condições semelhantes aos estudos realizados com atletas e/ou pessoas aparentemente saudáveis. O fato de não ter sido observado alterações entre as médias da PSE de cada sessão de fisioterapia, demonstra que a intensidade do exercício percebida pelo indivíduo não variou entre os atendimentos, mantendo sempre um mesmo padrão de esforço.

\section{Concentrações plasmática de lactato ([LA])}

A maioria dos estudos relacionados à produção de lactato e esforço físico utiliza protocolos em que a intensidade do exercício é aumentada progressivamente. A produção e o acúmulo de lactato estão diretamente relacionados à intensidade da atividade física exercida (17, 21, 26). No presente estudo, foi verificado aumento significativo das [LA] entre os tempos 0 e 45 minutos, evidenciando que a determinação da [LA] pode ser de extrema importância para a programação de uma reabilitação segura e eficaz, para pessoas com alguma doença associada, como a DMD (27).

Por outro lado, sabe-se que o treinamento promove redução da [LA] após cargas submáximas de trabalho, sendo que, quanto maior o nível de treinamento, menor a [LA] $(17,21,26)$. Contudo, vale ressaltar que foram realizados apenas 6 atendimentos fisioterapêuticos e que o tratamento não teve como objetivo o treinamento muscular. Pelo contrário, a fisioterapia teve como cuidado principal não fadigar o indivíduo. Isso justifica a ausência de variação das [LA] entres os 6 dias de atendimento fisioterapêutico.

Adicionalmente, apesar de valores numéricos mais baixos, a relação entre a PSE e as [LA] verificada no presente estudo foi similar às observadas em estudos com atletas e pessoas sem doenças acometidas $(17,21,24-26)$.

\section{Concentrações plasmática de glicose ([GLI])}

O consumo de glicose aumenta, durante o exercício físico, proporcionalmente à sua intensidade e duração. Em atividades prolongadas, o suprimento de glicose é caracterizado por ser quase constante durante a primeira parte, mantendo a concentração plasmática de glicose no nível de repouso ou levemente aumentada. Essas concentrações só começam a declinar mais tarde, em razão da perda da capacidade do fígado em produzir e liberar glicose $(19,22,28)$. No presente estudo, não foi verificada alteração significativa das [GLI] durante cada sessão de fisioterapia. Esses dados sugerem ocorrer tendência da manutenção dos níveis glicêmicos dentro de valores fisiológicos constantes durante a realização do tratamento proposto. 
Por outro lado, a alteração da [GLI] verificada durante as 6 sessões de atendimento fisioterapêutico pode ser justificada pela influência da dieta do indivíduo, pois não houve controle alimentar durante o estudo.

\section{Frequência cardíaca (FC)}

Sabe-se que a FC aumenta em proporção direta à intensidade do exercício $(18,20)$. No entanto, as alterações cardíacas em pacientes com DMD são frequentes, apresentando normalmente arritmias e elevação da FC de repouso. Dessa forma, a frequência cardíaca varia de acordo com o estágio da doença e do acometimento cardiovascular do paciente $(29,30)$, justificando a ausência de variação significativa da FC durante as sessões de fisioterapia, bem como da observação de uma relação diretamente proporcional entre FC e PSE, conforme verificado em estudos realizados com pessoas aparentemente saudáveis $(18,20)$.

Por outro lado, as variações das médias de FC verificada no decorrer das 6 sessões de fisioterapia não refletem em efeitos de condicionamento cardiovascular. Vale ressaltar que este não era um objetivo do tratamento proposto e ainda foram avaliadas apenas 6 sessões de fisioterapia.

\section{Pressão Arterial Sistólica (PAS) e Pressão Arterial Diastólica (PAD)}

A PAS tende a aumentar em proporção direta à intensidade do exercício. No entanto, estabiliza durante o estado estável do exercício submáximo, podendo até diminuir se esse período for prolongado. Mas, à medida que a intensidade da atividade volta a aumentar, a PAS também aumenta.

Durante as coletas de dados, as atividades realizadas pelo indivíduo eram de intensidade progressivamente aumentada a cada 15 minutos. No entanto, durante os 15 minutos de cada fase da sessão, a intensidade dos exercícios era estável. Isso pode justificar a ausência de diferença significativa entre os tempos 0', 15' e 30'. Nos últimos 15 minutos de atendimento fisioterapêutico, o indivíduo era posicionado sentado. E, apesar de todas as coletas, inclusive do último estágio da sessão, serem realizadas com o indivíduo em decúbito dorsal, a manutenção da postura sentada pode ter influenciado as medidas de PAS neste período. A manutenção da postura ortostática exige do sistema cardiovascular maior geração de força para enviar nutrientes necessários à musculatura ativa, o que requer maior aporte sanguíneo para manter a nova posição do corpo. Dessa forma, variáveis como FC e PA tendem a aumentar $(20,31)$.

Por outro lado, a PAD pouco se altera durante uma atividade de esforço, independentemente da intensidade $(20,31)$, conforme verificado no presente estudo. Adicionalmente, de acordo com o que foi mencionado anteriormente, pacientes com DMD possuem alterações cardiovasculares as quais podem influenciar nesta variável em estudo $(29,30)$.

\section{Frequência respiratória (FR)}

A FR aumenta durante o exercício de maneira proporcional às necessidades metabólicas do corpo, sendo regulada de forma a maximizar o desempenho $(17,18,20)$. Contudo, isso não foi verificado no presente estudo. A irregularidade da FR observada pode ser justificada pela realização de exercícios respiratórios ativos, que tinham como objetivo o aumento da capacidade pulmonar, reduzindo, dessa forma, a FR, entre os 15 e 30 minutos. Além disso, segundo Hahn et al. (32), pacientes com DMD apresentam fraqueza da musculatura inspiratória, o que leva a alterações da ventilação, redução da força muscular acessória e insuficiência respiratória crônica.

Diante do exposto, verificou-se que as variáveis fisiológicas avaliadas, como a PAS, a PAD, a FC e a FR, não aumentaram linearmente com o tempo, prejudicando a graduação da intensidade do exercício proposto como forma de tratamento, bem como da sensação de cansaço do sujeito do estudo. 
Por outro lado, as variáveis metabólicas, mais especificamente a [LA], variaram progressivamente, evidenciando aumento do trabalho muscular, diante do esforço realizado. No entanto, as [LA] e [GLI] foram verificadas por meio de procedimentos invasivos e que geram certo desconforto para o indivíduo. Além disso, são procedimentos mais caros e menos acessíveis aos pacientes que queiram monitorar a intensidade do tratamento fisioterapêutico por meio dessas variáveis. Já a PSE, verificada com a escala CR10 de Borg, aumentou linearmente com o tempo decorrido do atendimento fisioterapêutico em proporção direta com a [LA]. Esses achados evidenciam a sensação de cansaço do indivíduo e, consequentemente, o grau de intensidade da atividade exercida.

A escala CR10 de Borg é um método de fácil compreensão pelo paciente e também de fácil aplicação pelo terapeuta. Adicionalmente, ao contrário das outras variáveis analisadas, considera também fatores psicológicos do paciente, que são fundamentais para a aceitação e colaboração do tratamento proposto. Desta maneira, por não ter custos altos e não envolver procedimentos invasivos, a escala CR 10 de Borg pode ser utilizada em grande variedade de ambiente e em diversos níveis sociais.

Por fim, a partir dos resultados obtidos, sugere-se que a utilização da Escala CR10 de Borg para PSE, durante os atendimentos fisioterapêuticos em pacientes com DMD, pode ser de extrema importância para graduar o grau de cansaço do paciente, uma vez que a fadiga é o fator limitante no seu tratamento. Contudo, é importante salientar que estes dados são baseados em um estudo de caso e que novos estudos são necessários para a continuidade de investigação nesta área de atuação da fisioterapia.

\section{Referências}

1. Sussman M. Duchenne muscular dystrophy. J Am Acad Orthop Surg. 2002;10(2):138-51.

2. Deconinck N, Dan B. Pathophysiology of Duchenne muscular dystrophy: Current hypotheses. Pedriat Neurol. 2007;36(1):1-7.

3. Lankester BJA, Whitehouse MR, Gargan MF. Duchenne muscular dystrophy. Current Orthopaedics. 2007;21:298-300.

4. Voisin V, de la Porte S. Therapeutic strategies for Duchenne and Becker dystrophies. Int Rev Cytol. 2004;240:1-30.

5. Manzur AY, Kinali M, Muntoni F. Update on the management of Duchenne muscular dystrophhy. Arch Dis Child. 2008;93(11):986-90.

6. Anderson MS, Kunkel LM. The molecular and biochemical basis of Duchenne Muscular Distrophy. Trends Biochem Sci. 1992;17(8):289-92.

7. Roberts RG. Dystrophins and dystrobrevins. Genome Biol. 2001;2(4):REVIEWS3006.

8. Tyler KL. Origins and early descriptions of Duchenne muscular distrophy. Muscle Nerve. 2003;28(4):402-22.

9. Biggar WD, Klamut HJ, Demacio PC, Stevens DJ, Ray PN. Duchenne muscular dystrophy: current knowledge, treatment, and future prospects. Clin Orthop Relat Res. 2002;(401):88-106.

10. Evans NP, Misyak SA, Robertson JL, Bassaganya-Riera J, Grange RW. Dysregulated intracellular signaling and inflammatory gene expression during initial disease onset in Duchenne muscular dystrophy. Am J Phys Med Rehabil. 2009;88(6):502-22.

11. Escobar-Credillo RE, Miranda A, Lona S, Castillo M. Análisis del patron de interferencia en pacientes con distrofia muscular. Rev Neurol. 2004;39:517-20.

12. Frezza RM, Silva SRN, Fagundes SL. Atualização bibliográfica do tratamento fisioterapêutico das distrofias musculares de Duchenne e de Becker. Rev Bras Promoção da Saúde. 2005;18(1):41-9.

13. Bushby K, Bourke J, Bullock R, Eagle M, Gibson M, Quinby J. The multidisciplinary management of Duchenne muscular dystrophy. Curr Pediat. 2005;15:292-300. 
14. Cossu G, Sampaolesi M. New therapies for Duchenne muscular dystrophy: challenges, prospects and clinical trials. Trends Mol Med. 2007;13(12):520-6.

15. Palmieri B, Sblendorio V, Ferrari A, Pietrobelli A. Duchenne muscle activity evaluation and muscle function preservation: is it possible a prophylactic strategy? Obes Rev. 2008;9(2):121-39.

16. Grange RW, Call JA. Recommendations to define exercise prescription for Duchenne muscular dystrophy. Exer Sport Sci Rev. 2007;35(1):12-7.

17. Ribeiro JP, Yang J, Adams RP, Kuca B, Knutten HG. Effect of different incremental exercise protocols on the determination of lactate and ventilatory thresholds. Braz J Med Biol Res. 1986;19:109-17.

18. Weltman A, Weltman J, Rutt R, Seip R, Levine S, Snead D, et al. Percentages of maximal heart rate, heart rate reserve, and $\mathrm{VO}_{2}$ peak for determining endurance training intensity in sedentary women. Int J Sports Med. 1989;10(3):212-6.

19. Bosch AN, Dennis SC, Noakes TD. Influence of carbohydrate loading on fuel substrate turnover and oxidation during prolonged exercise. J Appl Physiol. 1993;74(4):1921-7.

20. Lambert EV, St Clair Gibson A, Noakes TD. Complex systems model of fatigue: integrative homoeostatic control of peripheral physiological systems during exercise in humans. Br J Sports Med. 2005;39(1):52-62.

21. Lima-Silva AE, De-Oliveira FR, Gevaerd MS. Mecanismos de fadiga durante o exercício físico. Rev Bras Cineantropom Desempenho Hum. 2006;8(1):105-13.

22. Lima-Silva AE, Fernandes TC, De-Oliveira FR, Nakamura FY, Gevaerd MS. Metabolismo do glicogênio muscular durante o exercício fisco: mecanismos de regulação. Rev Nutr. 2007;20(4):417-29.

23. Sveen ML, Jeppesen TD, Hauerslev S, Kober L, Krag TO, Vissing J. Endurance training improves fitness and strength in patiente with Becker muscular dystrophy. Brain. 2008;131(Pt 11):2824-31.

24. Borg GA. Psychophysical bases of perceived exertion. Med Sci Sports Exerc. 1982;14(5):377-81.

25. Borg GA. Escalas de Borg para a dor e o esforço percebido. São Paulo: Manole; 2000.

26. Greco CC, Denadai BS, Pellegrinotti ÍL, Freitas AB, Gomide E. Limiar anaeróbio e velocidade crítica determinada com diferentes distâncias em nadadores de 10 a 15 anos: relações com a performance e a resposta do lactato sanguíneo em testes de endurance. Rev Bras Med Esporte. 2003;9(1):2-8.

27. DeGroot DW, Quinn TJ, Kertzer R, Vroman NB, Olney WB. Lactic acid accumulation in cardiac patients performing circuit weight training: implications for exercise prescription. Arch Phys Med Rehabil. 1998;79(7):838-41.

28. Hargreaves M. Carbohydrate metabolism and exercise. In: Clarkson PM. Exercise and sport science. Philadelphia: Lippincott Williams e Wilkins; 2000.

29. Cox GF, Kunkel LM. Dystrophies and heart disease. Curr Opin Cardiol. 1997;12(3):329-43.

30. Finsterer J, Stollberger C. The heart in human dystrophinopathies. Cardiology. 2003;99(1):1-19.

31. Mendonça JT, Carvalho MR, Costa RK, Barroso RC, Santos JE, Tavares Filho SC. Reconstructive surgery of the aortic valve. Rev Bras Cir Cardiovasc. 2003;18(4):364-9.

32. Hahn A, Bach JR, Delaubier A, Guillou ARIC, Rideau Y. Clinical implications of maximal respiratory pressure determinations for individuals with Duchenne muscular dystrophy. Arch Phys Med Rehabil. 1997;78(1):1-6.

Recebido: 27/08/2008

Received: 08/27/2008

Aprovado: 02/10/2009

Approved: 10/02/2009 